\title{
USING THE DELPHI METHOD IN EVALUATIONS - INCORPORATING A FUTURE ORIENTED PERSPECTIVE IN EVALUATIONS
}

\author{
MICHAEL DINGES, ANNA WANG AND KLAUS SCHUCH \\ DOI: 10.22163/fteval.2020.469
}

\section{ABSTRACT}

$\mathrm{T}$

his article discusses the scope of use of the Delphi method in evaluations. It suggests that the Delphi method is a promising approach for validating the evidence gathered by incorporating a forward-looking perspective in the drawing of conclusions and in elaborating recommendations by making use of expert knowledge from different domains. Thereby, the Delphi method also facilitates information and engagement of relevant stakeholder communities in an ongoing evaluation process. Based upon the application of the method in the Evaluation of the Support Structures for Horizon 2020, EUREKA, COSME, EEN and ERA in Austria, the article demonstrates how the application of the Delphi method allows to analyse the degree of fit of an existing service portfolio against changing framework conditions and expected developments in the future.

\section{INTRODUCTION}

Evaluations of Ryl interventions face the challenge of transforming evidence on the performance of an intervention into recommendations on design options and broader policy recommendations in the observed domain. In the process of formulating recommendations, the core perspective of an evaluation changes: While the analysis of performance is predominantly backward oriented by considering the constituent actors and their changing contexts in an ex-post perspective, the process of formulating recommendations and design options requires careful consideration of the existing evidence combined with possible future developments of contexts and actors addressed by an intervention. Thus the perspective changes from retrospective (evidence-based answer to questions on 'what happened' and 'what worked well or not') to prospective ('what likely needs to be done to anticipate and successfully tackle future challenges').

While evaluations are usually categorized as being largely formative or summative (Chen 1996, Patton 1996), Edler et al. (2012) show in their empirical analysis that: 1) rather summative issues such as 'outputs, outcomes, and impacts', 'goal attainment and effectiveness', 'internal and external consistency' in evaluations are strongly linked to more formative topics such as 'policy/strategy development' and 2) this type of "holistic evaluations" seeks to understand and measure the programme

logic. This type of evaluation usually focuses on combining survey data with case studies, some (limited) network analysis and applies, as a consequence of the additionality assessment, some (limited) before/after group comparisons (ibid.).

However, even holistic evaluations performed today fall short in applying methods, which focus on (1) the possible future evolution of relevant contexts of an intervention as well as (2) presumed strategies of different types of actors towards the anticipated futures. Still, evaluation methods and monitoring practices are largely based on linear ideas and most evaluations are backward looking (ex post) despite the development and application of ex ante evaluation methods (Nieminen and Hyytinen, 2015). In particular, the application of methods, which can provide future oriented strategic decision support, are yet untapped in current evaluation practices of innovation policies.

Against this background we ask how a forward-looking perspective can be integrated in evaluations in a cost-efficient and effective manner? Nieminen and Hyytinen (2015) suggest that the essential characteristics of evaluation and foresight methodologies, complemented by dynamic system modelling as well as robust societal embedding, can provide both high-level 'visionary' inputs together with specific and detailed information for decision-making.

Taking up this perspective, we demonstrate how the Delphi method can be used as a tool not only for validating the evidence gathered, but also how to incorporate a forward-looking perspective in drawing conclusions and elaborating recommendations by making use of expert knowledge from different domains. The empirical basis for the discussion is the Evaluation of the Support Structures for implementing the European Framework Programme for Research and Innovation (H2020), EUREKA, COSME, EEN and ERA in Austria (Dinges et al. 2018) commissioned by the Austrian Federal Ministry of Science, Research and Economy in 2017.

We first provide a short conceptual outline on the scope and use of the Delphi Method as referenced in the academic literature. We then proceed to describe how the Delphi-Process was implemented in the Evaluation of the Support Structures for Horizon 2020 in Austria. We show how the findings of the Delphi process influenced the process of synthesizing evaluation results and how policy recommendations were shaped through the methodological design. In a final section we discuss how the Delphi process can make evaluations more sensitive towards changing framework conditions and uncertain futures, and which prerequisites for implementation have to be taken into account. 


\section{THE DELPHI METHOD}

The Delphi Method was originally developed by the RAND Corporation for technological forecasting and applying a systematic approach to the utilization of expert opinions. The Delphi Method attempts to make use of informed intuitive judgement (of experts) and derives its importance from the realization that projections into the future, on which policy decisions must rely, are largely based on the personal expectations of individuals rather than on predictions derived from a well-established theory (Helmer, 1967)!

In its original configuration, the Delphi Method sets out a process, in which the various opinions of several experts on a particular issue, are combined into a single combined position. It is a method for structuring a group communication process so that the process is effective in allowing a group of individuals, as a whole, to deal with a complex problem (Linstone and Turoff, 1975; Cuhls, Blind and Grupp, 2002). Key objectives of the Delphi Method were to reduce the negative effects of group interactions (Gupta and Clarke, 1996) and to obtain the most reliable consensus of opinion of a group of experts (Dalkey and Helmer, 1962). Key design elements of the Delphi Method are anonymity, iteration, controlled feedback and participating experts (Geist, 2009; Rowe and Wright 1999):

- The anonymity of the Delphi allows all panellists to be removed from pressures encountered in a face-to-face interaction. All ratings and comments are submitted anonymously, therefore, members can change their minds without feeling judged by others in the group (Rowe et al., 1991).

- Group members, or panellists, generate ideas and comments about the issue or problem from individual brainstorming. The researcher distils those responses and presents them to the panellists in the form of a survey for a second round of input. Then, the results and responses are presented to the group members in condensed form for comment. This process is being iterated up to four times.

- Controlled feedback occurs between iterations when the researcher uses qualitative data (e.g. comments, reasons for ratings, etc.) as a form of qualitative feedback. Controlled feedback presented in an organized format allows panellists to read, comment on, and critique all the facets of the issue virtually simultaneously between iterations (Rowe et al., 1991).

- Statistical group response consists of quantitative feedback (e.g. medians and interquartile ranges, or means and standard deviations) based on the numerical ratings of each item. After the final iteration, the ideas and opinions are listed along with the descriptive statistics of the ratings (Rowe et al., 1991).

Over the decades, a number of variations of the method have been developed, adapting the application in different ways, such as techniques to select participants, types of questions employed, tools used for the analysis of responses, and type of outcome sought (Hirschhorn 2019). The application possibilities for Delphi processes were also widened through advances in ICT support tools, allowing for example to implement real-time Delphis and experimentation by securing the anonymity of participants etc. Brady (2015) details that the Delphi Method has been used in an array of different contexts, where expert knowledge is needed to inform decision making or to understand a phenomenon in greater depth including in particular public policy, health care, education, and technological forecasts. In public policy, the Delphi Method seeks to provide policy makers with a better understanding of policy design and implementation (Alder and Ziglio, 1996; Linstone and Turoff, 1975).

\section{THE USE OF DELPHIS IN RGI POLICY AND RGI POLICY EVALUATION}

In the domain of Rgl policy, the focus of the use of Delphis changed in the last decades. Whereas in the 1970s and 80s, Delphi exercises and critical technology studies conducted in the US, in France and the Netherlands focused on predicting and controlling the future, new forms of Delphis have been developed that do not necessarily strive to achieve consensus on future forecasts, but rather to map the diversity of opinion in order to enable public policy to imagine alternative paths of future developments and to design public policy in a responsive manner towards these potential paths (Havas, Schartinger and Weber 2009). For example, the foresight study in support of future EU Research and Innovation Policy (BOHEMIA, Weber et al. 2018), aimed to support the deliberations on future proposals for a post-2020 Research and Innovation Framework. The BOHEMIA Delphi statements were not meant to provide a broad overview of 'all things to come' but started from scenarios on the Future for Research and Innovation Policies in Europe.

For monitoring and evaluation, the Delphi technique can be used in various evaluation tasks to anticipate possible futures, for instance by assessing the 'problem' a certain program aims to solve and forecasting future changes ${ }^{2}$. It is a particular useful method in judgment and forecasting of situations in which pure model-based statistical options are not practical (ibidem). Despite its potential usefulness, the Delphi Method has not been widely used in evaluations of R\&l policies and institutions.

In the following, we therefore make the case how the Delphi technique was used in the case of the evaluation of the Austrian support structures for Horizon 2020, EUREKA, COSME, EEN and ERA.

\section{THE APPLICATION OF THE DELPHI METHOD IN THE EVALUATION OF THE SUPPORT STRUCTURES FOR HORIZON 2020 IN AUSTRIA}

\section{SCOPE AND DESIGN OF THE EVALUATION}

The evaluation aimed to undertake a systematic assessment of the Austrian support structures and implementation of Horizon 2020 and ERA, as well as EUREKA, COSME, and the Enterprise Europe Network 
(Dinges et al. 2018). The evaluation focused on the assessment of support services provided by the Austrian Research Promotion Agency - Division of European and International Programmes (FFG-EIP), which is the national contact point for Horizon 2020 on behalf of the Austrian Federal Ministry of Education, Science and Research ${ }^{3}$. FFG-EIP pursues the following objectives:

- to support successful participation in Horizon 2020 by means of information and advice,

- to provide system-orientated support for Austrian Ryl organisations,

- to be an expert partner for European R\&l programmes and ERA developments, and

- to provide analysis on participation and to provide Rgl policy support.

Against these objectives of FFG-EIP, the evaluation commissioned by the ministry focused on the following areas:

- General evaluation of the implementation of the support structures for Horizon 2020: Are orientation and implementation of available formats and support services - within the allocated budget - suitable and appropriately implemented to achieve the desired effects of measures regarding output as efficiently and effectively as possible?

- Evaluation of specific services: By respecting observable and anticipated developments in the R\&I domain (including R\&I policy), are the service formats adequate and which improvements should be made?

- Action required and options for action: Which white spots can be encountered and which new and better approaches and tools should be established? According to which principles should these essentially be designed?

While having a predominantly summative and ex-post oriented character, the evaluation questions emphasized that also anticipated developments in the Ryl domain should be taken into account and that the adequacy of support formats needs to be assessed against current and future developments in the European and Austrian research and innovations system. Future developments, for example related to the European R\&l policy domain and in particular to the development of the new Framework Programme for R\&l, Horizon Europe, as well as the future shaping of the European Research Area, but also to the development of strategic capacities of Ryl actors, should be considered.

The evaluation approach was set up against an impact model, which linked actions of FFG-EIP with desired outcomes and impacts. For answering the evaluation questions, a mixed method evaluation design was chosen. It allowed for triangulation of quantitative and qualitative methods. Key components included online surveys with key stakeholder groups, interviews with a broad range of Ryl policy actors and stakeholders, focus group interviews, the Delphi Method, validation workshops, and critical commenting through an external advisory board.

Within this overall design, the two-stage online Delphi Method was chosen as a key component in the validation of evaluation results and the development of scenarios and policy recommendations by providing converging expert assessments on hypotheses and preliminary conclusions during the final phase of the evaluation.
The evaluation was performed from August 2017 until end of June 2018. Within this timeframe, the two rounds of the Delphi survey were operationalised from February until beginning of May 2018.

\section{SELECTION OF EXPERTS}

In order to benefit from a broad range of expert assessments on the support services and governance for Horizon 2020 in Austria, a sample of 190 experts were chosen due to their high knowledge of the evaluation subject at hand. They are formed from the following stakeholder groups: 1) senior university leadership (rectors and vice-rectors), 2) management of non-university research organizations, 3) management of enterprises that are highly active in Horizon 2020,4) policymakers, including delegates and experts of Austria to Horizon 2020 programme committees and representatives of relevant ministries, 5) representatives of national and regional funding agencies.

To ensure an adequate range of opinions, the experts were approximately equally distributed across the five stakeholder groups. In the first stage, 69 experts shared their assessment and were subsequently invited to the second-stage. 39 of these responded to the second round of the Delphi.

\section{IMPLEMENTATION OF THE DELPHI}

The Delphi surveys were part of the final phases of the evaluation, when other forms of data collection and analysis have had already been concluded. Thus, it was applied as a method of gathering expert assessments on specific hypotheses and conclusions derived from the evaluation in order to feed into a forward-looking development of scenarios and recommendations. Five overarching thematic areas were covered, each with their corresponding hypotheses and conclusions. These topics include:

1. the quality, speed, and transparency of communication and information on developments in European Rql,

2. the assumed necessity for strategically supporting companies, universities and research organizations in developing (pro-)active participation strategies for Horizon 2020,

3. the increasing demand for improved strategic support services in light of new thematic and organisational challenges posed by Horizon Europe,

4. the assumed importance of increased strategic multi-level coordination of stakeholders for European public-public and publicprivate partnership instruments,

5. the expected impacts of the new mission-oriented approach in Horizon Europe for national Ryl policies and governance.

Each topic was introduced by describing its context and importance. In addition, likely demands placed on R\&l governance and support services - based on expected developments in Austrian and European Ryl policy - were shortly mentioned. In the first stage of the Delphi, a set of three to five hypotheses were assigned to each topic. These hypotheses were based on the findings of the previous analytical evaluation steps 
and formulated as preliminary conclusions or recommendations to Rq। policymakers. The participating experts were asked to rate each quantitatively using a five-point Likert scale from "Yes, I fully agree" to "No, I strongly disagree". Additionally, experts were asked to give a qualitative statement explaining their rating for each. Table 1 Example of Delphi stage 1 questions provides an example of how a topic was presented, as well as how both open-ended and closed questions were utilized in the Delphi.

\section{Topic: Mission-orientation and preparation for Horizon Europe}

\section{Hypothesis:}

Horizon Europe will most likely be characterized by a stronger mission-orientation of R\&l policy. Thus, the new framework programme will have a set of pre-defined cross-thematic and cross-disciplinary R\&l goals for funding. These goals should have high societal impacts (e.g., at the interface of energy, environment, mobility, health, security, etc.) that should be measurably achieved in a relatively short time frame and carry with them significant value for society.

This new mission-oriented approach to R\&I policy demands: i) a common understanding of specific joint goals, ii) a strong alignment of European and national R\&l policies, iii) strong governance and strategic mission management on programme and project levels. Therefore, mission-orientation imposes high demands on the formulation, implementation, and support of R\&l policy.

\section{Please assess the following statements:}

Statement 1: Innovation is not sufficiently important in the European Research Area.

$$
\begin{array}{ll}
\mathrm{O} & 1-\text { No, I strongly disagree } \\
0 & 2 \\
0 & 3 \\
0 & 4 \\
0 & 5-\text { Yes, I fully agree }
\end{array}
$$

\section{Please explain your answer:}

Statement 2: In case there is a stronger mission-orientation on European level, Austria needs a national mission strategy, i.e., a with national stakeholders coordinated common approach.
O 1 - No, I strongly disagree
○ 2
○ 3
○ 4
O 5 - Yes, I fully agree

\section{Please explain your answer:}

Statement 3: With a stronger mission-orientation on European level, Austrian R\&l funding needs to be adjusted to align with European missions.
O 1 - No, I strongly disagree
○ 2
○ 3
○ 4
O 5 - Yes, I fully agree

\section{Please explain your answer:}

Statement 4: With a stronger mission-orientation on European level, the Austrian Research Promotion Agency needs to improve its support services in information provision, community management, and coordination.
O 1-No, I strongly disagree
○ 2
○ 3
○ 4
O 5 - Yes, I fully agree

Please explain your answer:

Table 1 Example of Delphi stage 1 questions 


\section{ANALYSIS OF RESULTS OF THE FIRST STAGE}

Overall, experts agreed moderately to strongly with the hypotheses of the first stage. Additionally, the use of free text answers for each statement under scrutiny allowed them to elaborate on their quantitative assessment. This feedback provided valuable input and explanations for (dis-)agreement and highlighted ideas for areas of improvement in Rql policy design. Thus, it can be argued that the first stage of the Delphi survey can be characterized as serving two key functions: i) validation of evaluation findings and preliminary conclusions, and ii) scoping forwardlooking options for Ryl policy design in response to expected changes at European and national RqI policy level.

The results of the first stage were compiled, analysed, synthesised and presented to the expert group during the second Delphi stage: First, the quantitative ratings were calculated for each question and summarized in a bar chart. Then the free text answers were analysed and summarized and, if relevant differences became apparent, differentiated by stakeholder groupings. The aim was to synthesize all answers into a 'story' to present the range of opinions, if and why the hypothesis was (in-)correct, and whether any important aspects were missing. The results of both the quantitative and qualitative analysis were then used to re-formulate hypotheses into conclusions by taking into account inputs and ideas raised by the experts.

\section{THE SECOND STAGE OF THE DELPHI}

In the second round of the Delphi survey, the respondents of the first round were confronted with the summative results of the first round in the forms of a graphical representation of quantitative assessments, the summaries of open-ended answers, as well as re-formulated conclusions, sometimes complemented by recommendations.

The formulation of new questions closely resembled those of the first stage by making use of both quantitative and qualitative assessment options: For each conclusion, participants were asked to give again a quantitative assessment on a five-point Likert scale and were invited to take the opportunity to comment.

\section{SYNTHESIS OF RESULTS}

The results of the second stage showed that expert opinions converged to a certain degree. Most of the responses displayed high agreement with our conclusions. The analysis and synthesis of results followed the same two-step method of summarizing quantitative results, drafting a summarized overview of qualitative responses, and, if necessary, reformulating conclusions while incorporating additional points raised by surveyed experts.

As a result of the two-stage process, the Delphi produced a set of validated conclusions that took into account anticipated demands on changes in policy orientation and governance associated with future developments at the level of national and European Ryl policy. The Delphi survey showed in particular, that the future role, functions and tasks of FFG-EIP do not only depend on the framework programme - they also depend on Austria's intended positioning vis-a-vis European R\&l policy.

\section{POLICY PATHWAYS FOR AUSTRIA IN VIEW OF HORIZON EUROPE}

Based on the results of the Delphi survey three alternative policy pathways for the future orientation of Austrian governance and support services for Horizon Europe were synthesized and finally discussed with Ryl policy-makers in Austria. These pathways were in particular developed in order not to limit the further design of the roles, functions and tasks of the FFG-EIP to rectify existing deficiencies or just to improve them against the backdrop of current tasks and challenges, but to take into account future challenges stemming from changing framework conditions such as the EC's proposal for the 9th European Framework Programme for Research and Innovation ("Horizon Europe") (see Dinges et al, 2018 for further details).

Our evaluative findings about the expectations of the Austrian Ryl community clearly showed that changes will be necessary in governance at national level and also at the level of the interface with European policy, as well as in the support services for the R\&I community. It became also clear that the extent of these changes depends on Austria's intended positioning regarding European R\&l policy. The three pathways reflect options for action at national level and the respective consequences that these would have for the support structures. They were framed as "food for thought" for further discussion in Austria, not least, also with regard to the development of a new R\&l strategy.

The three following scenarios/policy pathways were constructed:

1. "Enhancement in continuity" scenario: Incremental change

2. "Smart and proactive alignment" scenario: R\&l policy as a catalyst in the European multilevel system

3. "Distributed empowerment" scenario: Strengthening and crosslinking the RyI community

The central idea of the first scenario is that the well-established and largely well-functioning status quo is maintained and incrementally adapted to the requirements stipulated by the new tools and initiatives at European level, but without significant changes having to be made in Austria, e.g. in the area of governance. Consequently, elements of national strategy planning and prioritisation (e.g. with regard to Austrian participation in strategic partnerships) is negligible or limited to individual preferences of ministries.

In the second scenario, Austrian policy develops its role to that of a (pro)active contributor to European R\&l policy, and to make more targeted and effective use of the opportunities resulting from this for Austria ('smart alignment'). To be better able to positioning Austria as an integral element of the multilevel innovation ecosystem in Europe, Austria would need to implement a range of changes within the national system to achieve more intensive and more influential cooperation in the design and subsequently in the use of European R\&I policy. Specifying such a concept for a consistent Austrian positioning within the overall European R\&I policy would need to be a central element within the forthcoming Austrian R\&l strategy.

The third scenario sees the extension of participation and involvement in European R\&I policy, particularly by means of empowering the strategy and networking capabilities of R\&l actors themselves. This scenario assumes that organisations have an independent development strategy and ability to self-organisation in strong networks. However, for this policy-pathway also appropriate resources and capacities are requi- 
red, whose mobilisation needs to be supported by R\&l policy (e.g. by means of financial incentives, networking activities, information sharing, etc.). The experiences of other European countries show that there can be losers as well as winners in this empowerment process.

\section{DISCUSSION AND CONCLUSION: THE UTILITY OF THE DELPHI METHOD IN RGI POLICY EVALUATION}

As demonstrated by the case of the Evaluation of the Support Structures for Horizon 2020, EUREKA, COSME, EEN and ERA in Austria, the Delphi Method can be a useful tool for supporting the formulation of policy recommendations and pathways that consider challenges and potentials for re-orientation of R\&l policies that are associated with future developments.

The case reveals that the implementation of a Delphi in evaluation of Ryl policy must be well-timed in the overall research design, and best applied in the final phases of an evaluation with the aim to produce a set of validated, future-oriented conclusions and recommendations.

The combination of both a quantitative and a qualitative element in each question allows the Delphi Method to be particularly useful for the validation of evaluation findings while at the same time serving to gather expert ideas on improvements in Ryl policy design.

Special care needs to be taken in the selection of experts to ensure the reflection of a broad range of opinion and expertise, as this might have a high impact on the quality of results. Due to the efforts required for the analysis of qualitative data, the sample should be limited in size but include all relevant experts and stakeholder groups in an appropriate distribution. The relatively high statistical mortality in running at least two rounds of Delphi remains a problematic issue and needs to be considered respectively. In our case the statistical mortality was in total almost $80 \%$. This resulted in an above-average "survival" of stakeholder groups that are more used to formulate and to take positions (i.e. policy-makers and representatives of large companies and interest groups), which can lead to a distortion of perceptions.

To reduce statistical mortality, the effort for the addressed experts should be kept as limited as possible without destroying the overall narrative of the formulated hypothesis and the questions derived thereof. Otherwise the Delphi exercise would run risk to transform into a series of hardly connected and de-contextualized surveys.

Thus, it maybe would have been better in our specific case to confront the addressed experts from the outset with a couple of scenarios/ policy pathways to which the hypothesis should have explicitly referred to. Such a scenario-based approach, which is broken down to the level of specific questions, would probably have provided more clear narratives and orientation and kept the attention of the experts for a longer duration, thus reducing statistical mortality. The approach taken by us, however, was in the reverse direction: the alternative future policy pathways for the Austrian R\&I policy to approach the challenges of the next Framework Programme for Ryl were constructed after the Delphi and not used as input to the Delphi although ideas of such policy pathways were implicitly already available, but neither transparently formulated nor explicitly referred to.
Despite the challenge of statistical mortality, the Delphi Method is particularly useful in situations where a broad range of stakeholders might be affected differently or place different demands on R\&l policy design in response to expected changes. It is a structured means of interaction in an evaluation process, which also allows to present and discuss core findings of an evaluation with a broader community of stakeholders.

In a more general perspective, the Delphi Method can be seen as a stakeholder engaging method, which allows to 1) put stronger emphasis on changing framework conditions in the synthesis of evaluation results, 2) facilitates to incorporate ex-post findings with a more forward looking perspective, and 3) provides better legitimation of recommendations.

Compared with large, iterative stakeholder workshops, the Delphi Method in R\&I policy evaluation facilitates the formulation of targeted, forward-looking policy options and recommendations that synthesize the collective expertise and knowledge available in a more time- and costefficient manner. Also feedback from more remote respondents (in terms of geography) can be included, who would usually rather not come to a workshop organised in the capital city Vienna. The results of the Delphi survey can be further fed into the discussion process with the contractor and a number of core stakeholders where they can contribute to increase acceptance of evaluation results and increase understanding for possible policy options.

\section{REFERENCES}

Adler, M., and Ziglio, E. (Eds.). (1996). Gazing into the oracle. Bristol, PA: Kingsley.

Brady, S. R. (2015). Utilizing and Adapting the Delphi Method for Use in Qualitative Research. https://doi.org/10.1177/1609406915621381

Chen, H-T (1996). A comprehensive typology of program evaluation. Evaluation Practice, Volume: 17 issue: 2, page(s): 121-130. Issue published: June 1, 1996; https://doi.org/10.1177/109821409601700204

Cuhls, K., Blind, K. and Grupp, H. (2002). Innovations for our Future. Delphi '98: New Foresight on Science and Technology. Series: Technology, Innovation and Policy (ISI). Physica-Verlag.

Dalkey, N. and Helmer, 0. (1962). An experimental application of the Delphi Method to the use of experts. Prepared for the US Air Force Project Rand. Manuscript. RAND Cooperation.

Dinges, M. et al. (2018). Evaluierung der Umsetzung von H2020, EUREKA, COSME, EEN und ERA in Österreich. 10.22163/fteval.2018.291.

Edler, J., Berger, M., Dinges, M. and Gök, A. (2012). The practice of evaluation in innovation policy in Europe. Research Evaluation, 21 (3), 167-182.

Geist, M. (2009). Using the Delphi Method to engage stakeholders: A comparison of two studies. Evaluation and program planning 33(2):14754. DOI: 10.1016/j.evalprogplan.2009.06.006

Gupta, U. G., and Clarke, R. E. (1967). Theory and applications of the Delphi technique: A bibliography (1975-1994). Technological Forecasting and Social Change, Volume 53, Issue 2, October 1996, Pages 185-211. 
Havas, A., Schartinger, D. and Weber, M. (2010). The Impact of Foresight on Innovation Policy-Making: Recent Experiences and Future Perspectives. Research Evaluation, 19(2):91-104. D0I: 10.3152/095820210X510133

Helmer, 0. (1967). Analysis of the Future. The Delphi Method. Manuscript. The RAND Corporation.

Hirschhorn, F. (2019). Reflections on the application of the Delphi Method: lessons from a case in public transport research. International Journal of Social Research Methodology, 22:3, 309-322, D0I: 10.1080/13645579.2018.1543841.

Linstone, H.A. and Turoff, M. (1975). The Delphi Method: Techniques and Applications, Addison-Wesley Publ., Reading/Mass.

Nieminen, M., and Hyytinen, K. (2015). Future-oriented impact assessment: Supporting strategic decision-making in complex socio-technical environments. Evaluation, 21(4), 448-461.

Patton, M. 0. (1996). A world larger than formative and summative. EvaIuation Practice, Volume: 17 issue: 2, page(s): 131-144. Issue published: June 1, 1996; https://doi.org/10.1177/109821409601700205

Rowe, G. and Wright, G. (1999). The Delphi technique as a forecasting tool: issues and analysis. International Journal of Forecasting, 15 (1999) 353-375

Rowe, G.,Wright, G. and Bolger, F. (1991). The Delphi technique: a reevaluation of research and theory. Technological Forecasting and Social Change, 39(3), 235-251.

Weber et al. (2018). Transitions on the Horizon: Perspectives for the European Union's future research and innovation policies. Final report from project BOHEMIA - Beyond the horizon: Foresight in support of the EU's future research and innovation policy. Luxembourg: Publications Office of the European Union. doi: 10.2777/493572.

\section{AUTHORS}

\section{MICHAEL DINGES}

Austrian Institute of Technology

Giefinggasse 4, 1210 Wien

E: michael.dinges@ait.ac.at

\section{ANNA WANG}

Austrian Institute of Technology

Giefinggasse 4, 1210 Wien

E: anna.wang@ait.ac.at

\section{KLAUS SCHUCH}

Centre for Social Innovation

Linke Wienzeile 246, 1150 Vienna

E: schuch@zsi.at 